\title{
SIGNIFICADO CULTURAL DE LA MENSTRUACIÓN EN MUJERES ESPAÑOLAS
}

\section{CULTURAL MEANING OF MENSTRUATION AMONG SPANISH WOMEN}

\author{
Alicia Botello Hermosa* \\ Rosa Casado Mejía ${ }^{* *}$
}

\begin{abstract}
RESUMEN
Objetivo: Conocer qué significados culturales existen en nuestra sociedad respecto de la menstruación, desde la perspectiva de la enfermería transcultural y analizar si se han superado creencias falsas del pasado. Material y método: Estudio cualitativo con diseño basado en la Teoría Fundamentada y cuyas técnicas de investigación fueron la entrevista semiestructurada y los grupos de discusión. Participaron 47 mujeres de diferentes regiones de España. Como criterios de segmentación se establecieron la edad y el lugar de residencia. Como criterios de variabilidad el nivel educativo, madres o mujeres sin hijos/as y la profesión. Las categorías de análisis fueron la menarquia, menstruación, conocimientos sobre sus propios cuerpos y el género. Resultados: La menarquia es vivida de diferente forma según el grado de conocimiento previo; existencia de tabú en diferentes grados según la edad de la mujer, asociado a desconocimiento e ideas falsas; el traspaso de creencias populares erróneas, por transmisión oral, sigue presente. Conclusión: Los cuidados de enfermería transcultural deben ir encaminados a desterrar creencias falsas, utilizando talleres grupales que permitan erradicar creencias erróneas y ayuden a las mujeres a aclarar dudas que al respecto puedan tener.
\end{abstract}

Palabras clave: Menstruación, cultura, género, investigación cualitativa, enfermería transcultural.

\begin{abstract}
The objective of the research was to know what cultural meanings exist in our society regarding menstruation, from the perspective of transcultural nursing, and to analyze whether past false beliefs have been overcome. Method: Qualitative study with a design based on Grounded Theory and whose research techniques were semistructured interviews and discussion groups. 47 women from different regions of Spain participated. Age and place of residence were established as segmentation criteria and educational level, mothers or women without children and the profession were established as the variability criteria. The categories of analysis were menarche, menstruation, knowledge about their own bodies and gender. Results: The menarche is lived in a different way depending on the degree of prior knowledge; presence of taboo in different degrees depending on the age of the woman, associated to ignorance and false ideas; the transfer of erroneous popular beliefs, by oral transmission, is still present. Conclusion: Transcultural nursing care should be aimed at banishing false beliefs, using group workshops to eradicate erroneous beliefs and help women to clarify any doubts they may have.
\end{abstract}

Key words: Menstruation, culture, gender, qualitative research, transcultural nursing.

Fecha recepción: 24/11/14 Fecha aceptación: 29/03/17

\footnotetext{
*Antropóloga. Enfermera. Doctora en estudios de las Mujeres y de Género. Universidad de Sevilla. Profesora de la Facultad de Enfermería, Fisioterapia y Podología de la Universidad de Sevilla. España. Email: abotello@us.es

${ }^{* *}$ Antropóloga. Enfermera. Doctora en estudios de las Mujeres y de Género. Universidad de Sevilla. Profesora de la Facultad de Enfermería, Fisioterapia y Podología de la Universidad de Sevilla. España. Email: rcasado@us.es
} 


\section{INTRODUCCIÓN}

La menstruación, como fenómeno fisiológico, está imbuida en nuestra sociedad de fuertes connotaciones sociales y culturales que van más allá del proceso fisiológico en sí, por lo que podemos afirmar que es un proceso biopsicosocial. Respecto de este proceso, existen creencias culturales que influyen en lo que sienten las mujeres, en los modos de comportarse durante esos días, en fín, estereotipos que, incluso, influyen en las maneras de nombrarla o "no nombrarla" (1).

Desde la antropología feminista se ha teorizado y mostrado cómo la desigualdad social entre hombres y mujeres se proyecta a dos niveles: uno material, referido a las actividades socio-laborales en distintos ámbitos como el laboral o el doméstico y otro simbólico, centrado, sobre todo, en las representaciones asociadas al ámbito de la biología o fisiología humana, básicamente la reproductiva, donde la experiencia del cuerpo emerge como una esfera central. Pero, tanto en la antropología como en la teoría feminista general, la referencia principal ha sido siempre el cuerpo interno, principalmente el aparato reproductor, relegando todo lo concerniente a la imagen corporal, en su dimensión práctica y simbólica, a un lugar secundario en el análisis (2).

Es importante estudiar y comprender estos aspectos sociales y culturales, ya que se ha llegado a exponer que existe cierta incapacidad de tipo emocional, física y cognoscitiva, durante algunas fases del ciclo menstrual, para desempeñar tareas de responsabilidad. En este caso se intenta buscar un origen biológico a una serie de prejuicios que se emplean para intentar separar a las mujeres de ciertas esferas de poder y de control social (3).

De acuerdo con Aedo (4), "las construcciones y conceptualizaciones de género inciden en nuestras formas de pensamiento, nuestros métodos de conocimiento y el len- guaje con que nombramos y significamos la realidad". Así, como muchas de las actitudes existentes hacia el ciclo menstrual son negativas, en torno a la menstruación se crean muchos estereotipos e incluso discriminaciones hacia las mujeres durante estos días, que se visibilizan, por ejemplo, con ciertos miedos y prohibiciones relativas al uso del agua o preparación de alimentos (5).

No se puede afirmar que el ciclo menstrual continúa siendo un tema tabú en España, sin embargo, es patente el desconocimiento general existente sobre el mismo y en este sentido es urgente revisar cómo se han ido definiendo los periodos fundamentales de la vida de las mujeres, qué estereotipos existen, qué han representado para las propias protagonistas y cómo les ha afectado a su salud, todo con el propósito de detectarlas y proponer medidas para su corrección, que indudablemente pasarán por la educación para la salud (6).

Un fenómeno que nos permite entender esta problemática es la transculturalidad, que incide principalmente en los sistemas de creencias y valores de los seres humanos, y la enfermería trancultural (7) es un "área de estudio y trabajo centrado en el cuidado y basado en la cultura y en las creencias de la salud o enfermedad, valores y prácticas de las personas, para ayudarlas a mantener o recuperar su salud, hacer frente a sus discapacidades o a su muerte". De acuerdo con Leininger (8), su fundadora, su concepto de cuidados transculturales parte de la idea de que la salud y enfermedad, y por tanto los ciclos vitales femeninos, se presentan bajo un marco de referencia simbólico codificado, que da lugar a prácticas y comportamientos específicos, apoyados en mitos, creencias y costumbres arraigadas.

A esta premisa hay que unir la condición de género, que surge en estas situaciones, y da lugar a connotaciones diferentes respecto del cuidado humano, pues aquellos cuidados que están relacionados con el orden público o la aplicación de la fuerza física se le asignan 
al hombre, mientras que los cuidados relacionados con aquello que genera vida, como la fecundación y dar a luz, se le asignan a la mujer (9).

Leininger también afirmó que los cuidados culturales son los medios holísticos más amplios que permiten conocer, explicar, interpretar y predecir los fenómenos asistenciales de la enfermería para orientar a la práctica de esta disciplina (10), por ello en esta investigación se unen dos disciplinas complementarias, la Enfermería y la Antropología, porque mientras la Antropología analiza el significado cultural de diversas prácticas y puede comprobar la existencia de prejuicios culturales o creencias erróneas que repercutan negativamente en la salud, la Enfermería elabora programas específicos para intentar informar y erradicar esas creencias a los grupos de población diana o más vulnerables.

Según lo anterior, los objetivos de la investigación fueron: conocer los significados culturales asociados a la menstruación en mujeres españolas de diferentes generaciones, desde la perspectiva de la enfermería transcultural, y analizar si se han superado creencias falsas del pasado.

\section{MATERIAL Y MÉTODO}

Tipo de estudio: Investigación de tipo cualitativo (11), cuyo paradigma se centra en la descripción sistemática de la experiencia cotidiana (12), que utilizó como técnica de análisis la Teoría Fundamentada (13).

Participantes y criterios de segmentación: Se utilizó el muestreo teórico por ser apropiado cuando el principal propósito de la recolección de datos es generar una teoría sustantiva. Es continuo y gradual, guiado por la recolección de datos, el análisis y la interpretación a medida que se establece la teoría. Es especialmente coherente con los objetivos y técnicas de la teoría fundamentada. Así, par- ticiparon 47 mujeres españolas de las provincias de: Sevilla, Huelva y Badajoz. La edad se estableció como criterio de segmentación que permitió conformar 3 grupos: jóvenes (18-25 años, 26-35 años), mediana edad (3645 años, 46-55 años, 56-65 años) y ancianas (> 65 años). Otro de los criterios fue el lugar de residencia: ámbito rural o urbano, porque se pretendía comparar el nivel de creencias populares y estudiar si en el medio rural son más abundantes que en el medio urbano. Se utilizaron dos informantes por perfil.

Categorías de análisis: a) Menarquia: primera menstruación de la mujer, fenómeno fisiológico por el cual se hace ostensible que la niña ha llegado a la maduración física necesaria para procrear si así lo deseara; b) Menstruación: proceso fisiológico que tiene lugar cada mes de modo regular, aproximadamente durante treinta años de la vida de la mujer; c) Control y el saber sobre sus propios cuerpos: las mujeres que han sido excluidas mediante el sistema sexo-género de un protagonismo social, arrastran un desconocimiento de su propio cuerpo, sexualidad y reproducción, por lo tanto, es un dispositivo de poder del cual carecen y que tiende a mediatizar la relación que establecen con todo lo relacionado a su vida reproductiva (14); d) Género: organización social de la relación entre los sexos (15), papeles culturales asociados en cada época o sociedad a los sexos, por lo tanto, es un producto cultural que cambia con las sociedades y con el tiempo.

Criterios de variabilidad: a) Nivel educativo: sin estudios (ningún título académico), estudios medios (títulos académicos no universitarios) y estudios universitarios (diplomados y licenciados); b) Madres o mujeres sin hijos/ as: el planteamiento fue estudiar si las mujeres viven de forma distinta su ciclo vital siendo madres o mujeres sin hijos; c) Profesión: amas de casa o mujeres que trabajan fuera del hogar. 
Recolección de datos: Se realizaron 24 entrevistas semiestructuradas y 3 grupos focales (16). Las entrevistas semiestructuradas, que tuvieron una duración media de $45 \mathrm{~min}$, se realizaron en diferentes lugares de trabajo $\mathrm{y}$ en los domicilios particulares de algunas entrevistadas. En los grupos focales se resguardaron elementos mínimos de homogeneidad, de modo que se evitasen situaciones que dificultasen el discurso del conjunto del grupo, entre ellas, las diferencias de saber, de poder o de edad (17). Con ello, se pudieron rescatar las experiencias comunes y también se pudo asegurar la aplicación de los criterios de variabilidad citados. Cada grupo tuvo 7 participantes menos uno que tuvo 5. Las reuniones tuvieron una duración entre 65:10 hasta $80 \mathrm{~min}$, en horario de tarde. Con todos los grupos se siguió el mismo guión temático y para asegurar la recuperación íntegra de la información, las sesiones fueron grababas con videocámara, previo consentimiento informado. Así, el contenido de los grupos quedó reflejado en transcripciones literales, respetando tanto lo enunciado como el lenguaje analógico, tales como risas, silencios, entonaciones, etc. Para proteger el anonimato de las informantes cada entrevista fue codificada con la letra $\mathrm{M}$ de mujer, seguida del número secuencial de la entrevista o de grupo focal, el medio Rural o Urbano y la edad.

Análisis de contenido: Siguiendo los pasos propuestos por la Teoría Fundamentada o Grounded Theory $(13,18)$, posterior a la recolección de datos empíricos, se procedió a la codificación o análisis, es decir, segmentación, codificación y agrupación de los códigos en familia. Así, se pudo garantizar el movimiento "constante de idas y venidas" en el texto, lo que se define como análisis comparativo, hasta que se alcanzó la saturación teórica (12). El análisis de contenido de las entrevistas en profundidad se realizó mediante el software Atlas-Ti Versión 5.0 con licencia. Posteriormente se efectuó una triangulación de técnicas, comparando los análisis del dis- curso de las entrevistas semiestructuradas y de los grupos de discusión, para observar las similitudes en las categorías.

Aspectos éticos: Se siguió en todo momento la Declaración de Helsinki para hacer investigaciones éticas con seres humanos. Todas las participantes fueron informadas y se les pidió consentimiento informado para grabar las entrevistas respetando el anonimato y confidencialidad.

\section{RESULTADOS}

\section{Menarquia}

Respecto de esta primera categoría de análisis, en general las actitudes frente a cómo se vive la menarquia van a ser distintas, dependiendo del grado de información que la entrevistada tuvo cuando era niña, aunque en líneas generales se puede decir que las principales actitudes eran de timidez, vergüenza y sorpresa. La mayoría lo comentaba a una figura femenina de referencia, como las madres, hermanas o incluso abuelas:

"Yo creo que le daba como vergüenza a lo mejor de meterse en esos temas" (M6.R.46).

“Temas", según algunas entrevistadas, que no eran "bonitos para hablar", es decir, que tenían una carga de misterio y suciedad. Parece que no se concebía la menarquia y la menstruación como algo natural y fisiológico y en la actualidad las niñas siguen ocultando este hecho a sus madres, con lo que el tabú sigue existiendo:

\footnotetext{
"Es que era una cosa que ni se lo decía ni se lo escondía, sino que me ponía y ya está, no, que no iba diciendo "me he puesto mala" ni tampoco se lo ocultaba, te pones y ya está, tampoco había que hacerle una fiesta. Bueno, tabú... no es que fueran tabús pero que no eran temas como bonitos de hablar" (M6.R.46).
} 


\section{Menstruación}

Como se observa en los testimonios respecto de la segunda categoría de análisis, a la mujer se le enseñaba el hecho de ocultar la menstruación, viéndolo como algo sucio y negativo, en vez de un proceso fisiológico positivo. Existía transmisión de conocimientos de madres a hijas y el papel ejercido por las abuelas se repite en muchas informantes:

"Porque además, yo que sé... el tema de llevar a tu hija al ginecólogo, eso era impensable que mi madre en mi época me llevara a mí al ginecólogo" (M6.R.46).

"Es que, vamos, que yo pienso llevar a mi hija al ginecólogo en cuanto que sea mujer, vamos. Para que vean que no tiene ningún nodulito ni..., o yo que sé, pa que vean que todo es normal. No sé cómo tendrá que estar, pero vamos que, que esté normal” (M6.R.46).

"A mí me preparaban un neceser para meterlo en la maleta con una compresa, una toallita limpia, otras braguitas por si se manchaban... esa costumbre yo la sigo hoy en día y llevo lo mismo" (M7.G2.U.22).

"Mi abuela me compraba las bragas de algodón nuevas y me las metía en la maleta del colegio por si me manchaba" (M6.G2.U.25).

"Sí, con mucho cuidado... yo a mi hija de esas cosas no le he hablado... quizás por cortedad... ella me dijo un día jmamá ya me he puesto mala! Y entonces yo ya le dije que tenía que tener cuidado y ya la preparé... pero yo explicarle no... Yo le dije que no se bañara y que había que tener mucho cuidado" (M11.R.84).

En la investigación se ha evidenciado que en nuestra sociedad la menstruación como fenómeno fisiológico está imbuida de fuertes connotaciones sociales y culturales que van más allá del proceso fisiológico en sí. Este proceso biológico sigue teniendo hoy en día cierto halo de tabú, aunque no tan marcado como la menarquia. Además se aprecia, por los testimonios, que hoy en día hablar de la menstruación está más normalizado, quizás por la influencia de los medios de comunicación y la publicidad de los productos relacionados (compresas, tampones, salva slip, toallas):

\begin{abstract}
"Nosotras no hablábamos de esto con nadie con nadie, porque era un tema tan intimo, tan intimo... ahora veo que las niñas lo hablan con cualquiera... yo creo que se ha pasado de un tema a otro... no es que yo considere que es un tema tabú, pero es como cuando quieres hacer una necesidad fisiológica que no se te ocurre comentárselo a nadie, esto igual, es un tema intimo" (M4.G1.U.52).
\end{abstract}

"A mi hermana no le contaba nada, pero a mí, como era la que llevaba el tejemaneje de todo, me lo contaba, decía que eso era una cosa que había que tener mucho cuidado, porque cuando faltaba se quedaban las niñas embarazadas, que no se podía si algún hombre me llamaba por algún sitio que nunca le hiciera caso, ni aunque me diera dinero ni aunque me diera, tú sabes... y luego eso, lo del plan de la ropa” (M22.U.77).

La menstruación ha sido vivida por muchas mujeres como experiencia traumática, debido a la falta de información que se comentaba anteriormente con respecto a la menarquia. Asimismo, en la presente investigación se ha podido constatar la existencia de creencias erróneas relacionadas con la menstruación en el discurso de las mujeres, que ellas mismas han empezado a ponerlas en duda, cuando ellas indican "por su propia experiencia”. Creencias cuyo origen está en la consideración de la sangre de la menstruación como impura, tóxica, sucia, algo por tanto de lo que avergonzarse y tener que ocultar: 
"Lo del tampax era ponértelo a escondidas $y$ esconder las cajas porque ya no eres virgen. Cuando yo tuve la endometriosis le echaron la culpa a los tampax. Pero vamos, no se les puede culpar porque ellas mismas eran ignorantes, porque era un tema tabú, había que ocultarlo y no se decía nada" (M4.G1.U.52).

"Yo sí, yo no podía tocar nada; yo lo viví en primera persona, era una cosa demencial en ese sentido. No había información ni donde verlo, no es como ahora con internet. Yo tengo 47 años, los años 70 fueron mi pubertad, mi juventud, y es que en las bibliotecas municipales tampoco habia cosas de mujeres, y es que no había forma de documentarte. Yo me acuerdo que iba a la biblioteca municipal para ver que órganos tengo, cómo funcionan... iy no había ni un libro, pero ninguno!... pero es que las monjas tampoco sabian nada; las cosas que sabemos es eso, por pura experiencia, en ese sentido los primeros años igual que mi madre, hasta que más tarde te vas informando y bueno... por esa parte superas los traumas como los superamos todos" (M6.G1.R.47).

\section{Control y el saber sobre sus propios cuerpos}

Se trata de relatos sobre sus propios cuerpos en los que introducen otros niveles de muchísima importancia: la necesidad que tenían las mujeres de aprender, de conocer su propio cuerpo, entendiendo por ello la fisiología del ciclo menstrual, de los embarazos, de los partos, en general de los aspectos relacionados con la salud reproductiva. Un deseo que se ve claro también en el testimonio sobre su adolescencia de la siguiente informante:

"Estar en un pueblo de Extremadura en plena represión franquista en un pueblo alejado de la capital, en una de las regiones más atrasadas de España, ipues tú te puedes imaginar! Yo leía a Bécquer, a Machado... tú leías yo que sé ; a Ba- roja! y decías, este mundo no es el mío y esto que estoy viviendo no es realmente lo que yo necesito; sabes, son descubrimientos míos; a través de la literatura yo me realicé, conocí la importancia de la mujer, lo que podía aportar la mujer por el hecho de serlo, que nosotras creábamos" (M7.R.60).

Por tanto, podemos afirmar que las mujeres han tenido y siguen teniendo un gran descontrol y desconocimiento sobre sus propios cuerpos.

\section{Género}

La menstruación está imbuida de fuertes connotaciones sociales y culturales que van más allá del hecho fisiológico y que están relacionadas con la ideología de género. "Yo por ejemplo con mi hija que también le lle-
gó con 11 años yo he intentado quitarle todos
los traumas, todos los tabús, todas las cargas".
(M4.G1.U.52)

"La verdad es que era tremendo, cómo se tenía oculto como si fuese algo malo o motivo de vergüenza. Yo a mis hijas les he quitado todas las cargas, antes de que se desarrollasen les expliqué en qué consistía, que podían hacer su vida con total naturalidad, que era algo fisiológico y que no habia motivo para avergonzarse ni ocultarlo, que era un proceso cíclico del cuerpo de la mujer y que incluso se podían sentir orgullosas, eso era señal de que todo en sus cuerpecitos funcionaba bien y que en el futuro cuando fuesen más mayores podrían tener hijos”. (M3G3.U.48)

El término género, como constructo social que pone de manifiesto las convenciones culturales, los roles y los comportamientos sociales que diferencian a las mujeres y a los hombres; por tanto, intenta distinguir el sexo biológico de la forma en que la sociedad construye el «ser hombre» o «ser mujer». 


\section{DISCUSIÓN Y CONCLUSIÓN}

Lo que se destaca y se considera muy importante es que estas creencias erróneas y negativas continúan arraigadas en muchas mujeres, y que están basadas principalmente en la falta de información objetiva y veraz $(5,6)$.

El saber sobre sus propios cuerpos está inspirado en la premisa de Foucault (14) que confiere al saber una forma de poder $y$ de control que ejercen aquellos/aquellas que lo detectan y que se activa en las relaciones de poder, es un dispositivo de poder del cual carecen y que tiende a mediatizar la relación que establecen con todo lo relacionado a su vida reproductiva.

En los testimonios se aprecia la existencia de tabú en diferentes grados según la edad de la mujer, es decir, a más edad más tabú. En muchas ocasiones este tabú lleva asociado desconocimiento e ideas falsas. Pero el que se hable con otras personas, siempre cercanas, como en el caso de lo que refiere la informante, no significa que la calidad de la información que tienen las niñas sea la correcta (19).

Esta falta de formación específica sobre la menstruación genera inseguridad y las lleva a creer en la información informal que reciben por amigos o familiares, es lo que se conoce como conocimientos entre iguales (20), resultando que esta información entre iguales no es siempre de la calidad y rigor suficientes por lo que se pueden seguir transmitiendo estereotipos e ideas erróneas asociadas a impureza e inferioridad (21).

Para autoras como Sau (22), el ciclo menstrual es un hecho femenino, y como la mujer es el sexo dominado, los mitos y los tabúes, es decir, lo prohibido, lo peligroso, no hacen sino manifestar esta situación en sus formas de presentarse.

Con respecto a nuestro objeto de estudio, las actitudes negativas hacia la menstruación pueden llevar a actitudes negativas hacia las mujeres en general, pudiendo afectar también a la imagen corporal de la mujer y a su autoconcepto ya que la menstruación y el ciclo menstrual tienen en la mayoría de las sociedades connotaciones de suciedad e impureza $(22,23)$.

Las principales conclusiones del estudio son:

- Las creencias populares relacionadas con la menstruación han sido origen de fuertes desigualdades sociales y culturales entre hombres y mujeres. La diferencia biológica del ciclo menstrual se convierte de esta forma en una desigualdad social y cultural, ya que durante esos días las mujeres están sometidas a prohibiciones y exclusiones estratégicamente pensadas. A su vez, la desigualdad que supone la menstruación conlleva la discriminación de la mujer menstruante, prohibiéndosele participar en muchos actos y actividades tanto familiares como comunitarias.

- La menarquia es vivida de diferente forma según el grado de conocimiento previo que se tuviese cuando niña. A más conocimiento del hecho, más naturalidad y tranquilidad, y al contrario, a menos información, es vivida como una experiencia más desagradable.

- La menstruación se oculta en muchas ocasiones, ya que se vive como algo motivo de vergüenza y suciedad, incluso de impureza y toxicidad.

- El traspaso de creencias populares erróneas, por transmisión oral, sigue presente.

- La Enfermería transcultural tiene un papel muy importante en la educación para la salud sobre el hecho fisiológico de la menstruación y desde la perspectiva social y cultural, para desmontar creencias culturales erróneas sobre la menstruación que todavía existen y que afectan a la salud de las mujeres, así como para aclarar dudas al respecto en niños/as y adolescentes. 


\section{REFERENCIAS}

1. Botello-Hermosa A. La menarquia: influencia de las creencias populares y la cultura en la salud. Enferm Comun [Internet]. 2013 [citado 15 feb 2014]; 9(2). Disponible en: http://www.index-f. com/comunitaria/v9n2/ec9201r.php

2. Esteban ML. Antropología del cuerpo: género, itinerario, identidad y cambio. Barcelona: Bellaterra; 2004. 263 p.

3. Gómez J, Martínez JM. Psicobiología del ciclo menstrual. Madrid: Universidad Nacional de Educación a Distancia; 1991. $172 \mathrm{p}$.

4. Aedo MT. Género e investigación en Salud. Cienc. enferm. 2010; XVI(3): 7-8.

5. Botello-Hermosa A, Casado R. Miedos y temores relacionados con la menstruación: Estudio cualitativo desde la perspectiva de género. Texto contexto-enferm. 2015; 24(1): 13-21.

6. Botello-Hermosa A, Casado R. Estereotipos de género con respecto a las etapas reproductivas de las mujeres y sus implicaciones en la salud. Matronas profesión. 2016; 17(4): 130-136.

7. Leininger M. Cuidar a los que son de culturas diferentes requiere el conocimiento y las aptitudes de la enfermería transcultural. Cultura de los cuidados. 1999; Año III(6): 5-12.

8. Leininger $\mathrm{M}, \mathrm{McF}$ arland $\mathrm{Mr}$, editores. Transcultural nursing: concepts, theories, research and practice. $3^{\mathrm{a}}$ edición. Toronto: McGraw-Hill; 2002. p. 77-98.

9. Chávez R, Arcaya M, García G, Surca T, Infante M. Rescatando el autocuidado de la salud durante el embarazo, el parto y al recién nacido: representaciones sociales de mujeres de una comunidad nativa en Perú. Texto contexto-enferm. 2007; 16(4): 680-687.

10. Barco V, Cruz S, Rodríguez Z, Herrera D. Gestión del cuidado desde una perspectiva transcultural. Rev Cubana Enfermer [In- ternet] 2011 Mar [citado 5 jun 2014]; 27(1): 57-65. Disponible en: http://scielo. sld.cu/scielo.php?script=sci_arttext\&pi$\mathrm{d}=$ S0864-03192011000100007\&lng=es.

11. Ulin P, Robinson E, Tolley E. Investigación aplicada en salud pública: métodos cualitativos. Washington (DC): Organización Panamericana de la Salud; 2006. 286 p.

12. Vivar C, Arantzamendi M, López-Dicastillo O, Gordo C. La teoría fundamentada como metodología de investigación cualitativa en enfermería. Index Enferm. 2010; 19(4): 283-8.

13. Glaser B, Strauss A. The discovery of grounded theory: strategies for qualitative research. New York: Aldine de Gruyter; 1967. $271 \mathrm{p}$.

14. Rosales R. Trabajo, salud y sexualidad. Las cargas de trabajo laborales y reproductivas en la salud de las mujeres. Barcelona (Es): Icaria; 2002. 303 p.

15. Scott J. Gender: A Useful Category of Historical Analysis. The American Historical Review.1986; 91(5): 1053-1075.

16. García M, Rodríguez M. El grupo focal como técnica de investigación cualitativa en salud: diseño y puesta en práctica. Aten Primaria. 2000; 25(3): 181-6.

17. Murillo S, Mena L. Detectives y camaleones: el grupo de discusión: una propuesta para la investigación cualitativa. Madrid: Talasa; 2006. 166 p.

18. Soãres S, Leite J, Erdmann A, Prochnow A, Stipp M, García V. La teoría fundamentada en datos. Un camino a la investigación en enfermería. Index Enferm. 2010; 19(1): 55-9.

19. Botello A, Casado R. El tabú de la menarquia desde la perspectiva de género en salud. Aportaciones a la Investigación sobre Mujeres y Género. I + G 2014. V Congreso Universitario Internacional Investigación y Género; 3 y 4 Jul 2014; Sevilla: Universidad de Sevilla; 2014. p. 367-377;

20. Louis V. Everyday Discourses of Menstruation: Cultural and Social Perspecti- 
ves. McMillan; 2016. 213 p.

21. Orringer K, Gahagan, S. Adolescent girls define menstruation: a multiethnic exploratory study. Health Care Women Int. 2010; 31(9): 831-847.
22. Sau V. Reflexiones feministas para principios de siglo. Madrid: Horas y horas; 2000. 236 p.

23. Valls-Llobet C. Mujeres, salud y poder. Madrid (Es): Cátedra; 2010. 424 p. 